\title{
Treatment of congenital and juvenile cataract with intraocular lens implants (pseudophakoi)
}

\author{
C. D. BINKHORST* AND M. H. GOBIN† \\ *Terneuzen, Netherlands, and $\dagger$ Antwerp, Belgium
}

Although surgical and optical techniques are continually improving, the treatment of cataract in infancy and childhood is still complicated by such problems as amblyopia, squint, and monocularity. This holds true for traumatic cataract in early life and also for congenital and early acquired endogenous cataract.

We have already reported the results of treating unilateral traumatic cataract in childhood with intraocular lens implants (pseudophakoi), mainly irido-capsular lenses of our own design (Binkhorst, 1967; Binkhorst and Gobin, 1964, 1967; Binkhorst, Gobin, and Leonard, i 969 ). In the past 6 years we have also treated several children with congenital and juvenile cataract, most of them by the same method of irido-capsular fixation.

Pseudophakos implantation is not indicated in every such case; the child's age and the functional status of the eye have to be carefully considered. The anatomy and the "surgical misbehaviour" of the very young infant eye may be contraindications for the procedure, and eyes with total or subtotal cataracts that have to be operated upon at a very early age are not generally suitable. The youngest child to receive a lens implant was 8 months old, and the eldest I I years old. The best anatomical results are obtained from the age of I year onwards.

Functional status is influenced by such criteria as the density of the cataract (complete, nearly complete, incomplete), the presence of associated anomalies (microphthalmos, nystagmus, abnormal electroretinographic activity), the presence of squint, and the visual acuity. In older children the visual acuity should be determined repeatedly with care and patience under varying conditions of light and pupil size, with and without spectacle correction, after the child has been trained to cooperate in Snellen's E test. Very young children may need to be admitted to hospital for observation so that the visual performance of each eye can be assessed accurately.

The results in seventeen children are discussed below. The follow-up period in a few is long enough to list these results as definitive, but in others our report is merely preliminary. In all of them the pseudophakos is well tolerated, the pupil is clear, and there have been no complications.

\section{Group I Unilateral cataract}

Cases I to 9 were suffering from dense, total or subtotal cataract in one eye (Table I, overleaf). 
Unilateral cataract is usually detected because of associated anomalies. All of these nine patients had nystagmus, there was microphthalmos in five, and the affected eye was usually squinting. In Case 5 there was intermittent deviation and the prism test was positive. In every case, on occlusion of the normal eye, visual acuity proved to be nil; this was most probably due partly to cataract and partly to amblyopia.

Surgery of unilateral cataract is justified only when the child will benefit from it; for example, visual acuity may be improved, but this can only be achieved and maintained by energetic occlusion therapy. In all the children in our series the visual acuity improved, even if there was microphthalmos. Although we have not so far demonstrated visual acuity better than $0 \cdot 0 \mathrm{I}$ to $0^{\circ} \mathrm{I}$, all the children behaved as though they could see better when the normal eye was occluded. Occlusion was readily accepted by those who did not accept it before surgery, the common symptom of light sensitivity having disappeared. Nystagmoid movements were favourably influenced by the lens implantation, but there was no improvement in squint. In two cases the pseudophakos was implanted for cosmetic reasons. The full history of a case of unilateral cataract is given below.

CASE 5

A girl aged 2 years had a dense nuclear and lamellar cataract in her microphthalmic right eye. There was a very slow searching and partially latent nystagmus. Artificial mydriasis did not affect fixation. An intermittent convergent squint was present. Extracapsular extraction and implantation of a pseudophakos were both performed in one operative session. The patient's cooperation in the occlusion of the left eye was excellent.

Her present state ( $3 \frac{1}{2}$ years after implantation) is shown in Fig. I. The pseudophakos is well tolerated, the pupil is clear, and the visual acuity of the operated eye is 0.08 . The visual acuity of the fellow eye is 0.5 . There is an intermittent convergent squint and latent nystagmus. Simultaneous perception and fusion are present, the latter without amplitude.

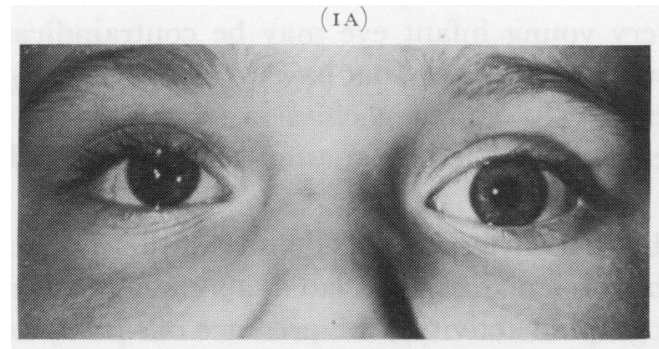

FIG. I Case 5. Unilateral cataract in microphthalmic right eye treated with irido-capsular pseudophakos implantation at the age of 2 years. Visual acuity 0.08. Simultaneous perception and fusion (without amplitude)

\section{Group II Bilateral subtotal cataract}

Cases 10 to 17 are shown in Table II (overleaf). Implantation was performed in one eye in Cases I I, I 2, I3, 14, and I5, and in both eyes in Cases I0, I6, and I 7 .

The cataract was discovered at varying stages for various reasons; nystagmus and squint led, in some cases, to its discovery at an early age. If no such signs were present the children were brought for examination because of apparently poor visual acuity at from 3 to 8 years of age. Microphthalmos was not seen in this group. 
w

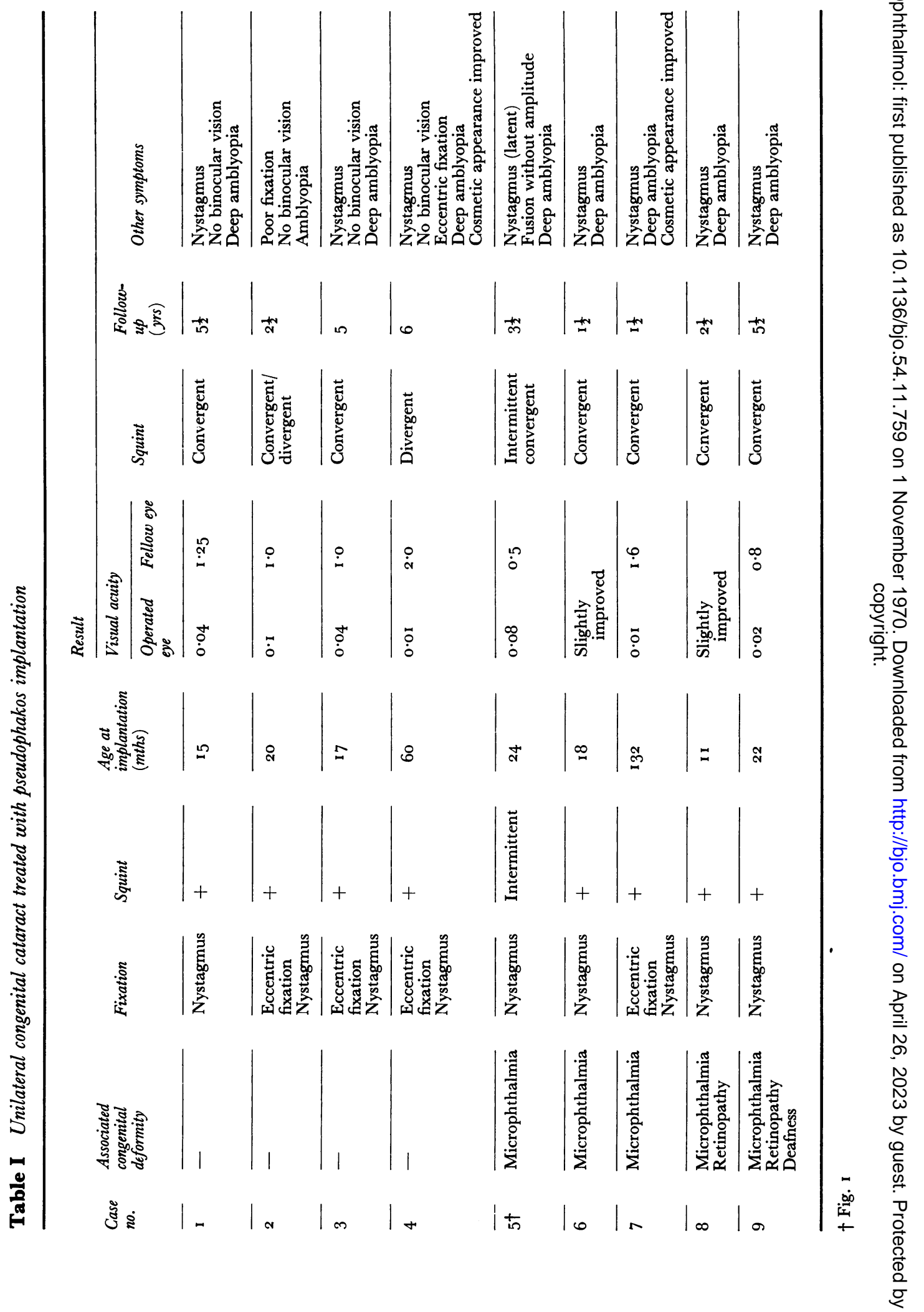




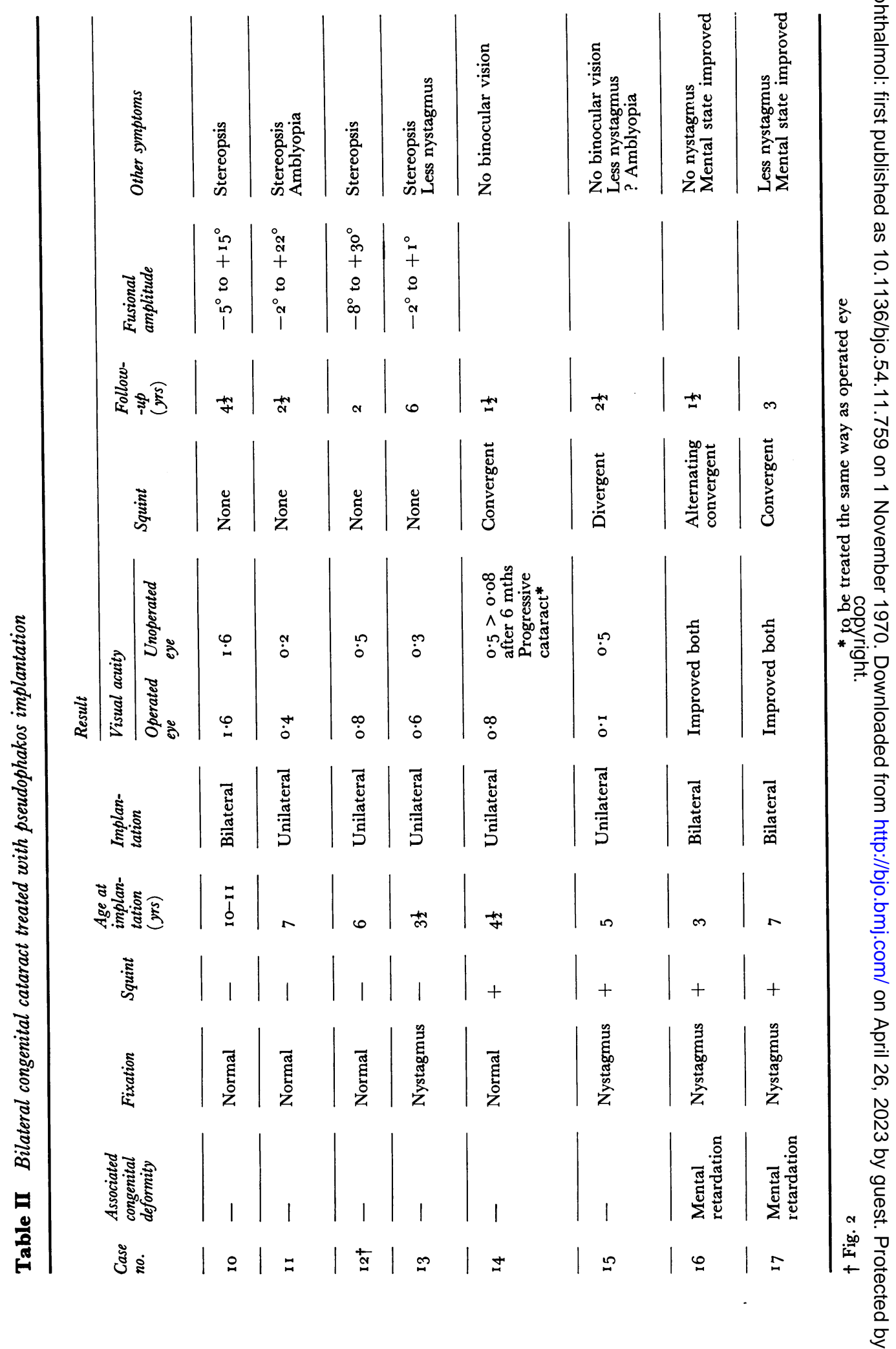


After surgery, the visual acuity, either measured by Snellen's E-test or estimated by the child's behaviour, improved markedly in every case. Nystagmus decreased and fixation improved. Two children with mental retardation developed well after surgery (Cases I6 and 17 ).

Three children had no squint before treatment (Cases I0, I I, and I 2). In Case io we twice had to deal with breakdown of fusion, but finally succeeded in restoring full binocularity; this 8-year-old boy developed unequally dense cataracts in both eyes. After cataract extraction in the worse eye and the fitting of a contact lens, this eye developed a convergent squint which was cured by pseudophakos implantation. A few months later, however, decreasing visual acuity of the fellow eye induced a convergent squint of this eye. Again the squint was cured, this time by cataract extraction and implantation of a pseudophakos. Since then this bilaterally pseudophakic boy has had full binocular vision. Cases I I and 12 had the worse eye only operated on. Care was taken to establish favourable anatomical and optical conditions, viz. a clear pupil, and the implantation was carried out in the shortest possible time. In both cases we succeeded in preventing breakdown of fusion.

In four children in this group (Cases 14 to 17 ), squint was present before treatment and, except in Case I 4, it was accompanied by nystagmoid movements of the squinting eye. In none of these children was lens implantation, either unilateral or bilateral, followed by a cure of the squint and the establishment of binocular vision, either spontaneously or after muscle surgery. It must be stated, however, that the conditions were unfavourable. In Case 14 more than 4 years elapsed between the first appearance of the squint and the implantation. In Case I 5 nystagmus, and in Cases 16 and 17 nystagmus and mental retardation, complicated the squint. In Case i 3 there was nystagmus without squint.

The superiority of pseudophakic treatment is clearly shown in this group. In Case ro implantation of a pseudophakos cured a convergent squint that had been started off by fitting a contact lens, and in Cases I I and I 2 implantation prevented breakdown of fusion. For the pseudophakic correction to be successful, however, aniseikonia and prismatic imbalance must be absent.

Extracapsular cataract extraction and implantation of the pseudophakos at the same operative session ensure favourable conditions for fusion. Great attention should be paid to signs pointing to a breakdown of fusion and thus to a squint, either of the operated eye (if visual acuity is not re-established quickly) or of the fellow eye (if visual acuity is too far below that of the operated eye).

Long-term postoperative pleoptic treatment is much more acceptable to a child wearing a lens implant than to one who has to cope with a high-powered spectacle correction or with a contact lens.

Another advantage is that the use of a lens implant greatly reduces feelings of inferiority. If a squint develops as a result of cataract, prompt pseudophakic treatment of this eye offers a fair chance of relieving it.

Such cases may be compared with those in which unilateral traumatic cataract causes a squint to develop (Binkhorst and Gobin, 1967; Binkhorst, Gobin, and Leonard, 1969).

An important feature of pseudophakic treatment, clearly demonstrated in Cases I I and 12, is that the implantation of a pseudophakos into the eye with the poorer visual acuity gives not only better visual acuity, but also binocular vision. In this way the surgical risk is limited to one eye only, whereas to achieve fusion by correcting the aphakia with a contact lens or with spectacles, would require bilateral surgery with the further risk of breakdown of fusion through prismatic imbalance. 
The following case history is a typical example of bilateral congenital cataract treated with a pseudophakos implantation in one eye.

CASE I 2

A boy aged 5 years had reduced visual acuity due to bilateral cataracts of the lamellar and anterior polar types. In favourable lighting the visual acuity was 0.3 in the right eye and 0.5 in the left. The eyes were straight. Extracapsular cataract extraction and implantation of a pseudophakos into the right eye were performed at one operative session. Occlusion of the left eye was then carried out each afternoon. The visual acuity of the right eye is now gradually improving and there are no oculomotor problems.

His present state (2 years after implantation) is shown in Fig. 2. The pseudophakos is well tolerated, and he is able to attend school. The visual acuity of the operated eye is 0.4 , and 0.8 with -2 D. cyl., axis $85^{\circ}$. That of the left eye is 0.4 , and 0.5 with -2 D. sph. The eyes are straight. Fusional amplitude ranges from $-8^{\circ}$ to $+30^{\circ}$. The synoptophore stereo test and fly stereo test are positive. The Wirt stereo test is $0 \cdot 3$.

$(2 \mathrm{~A})$

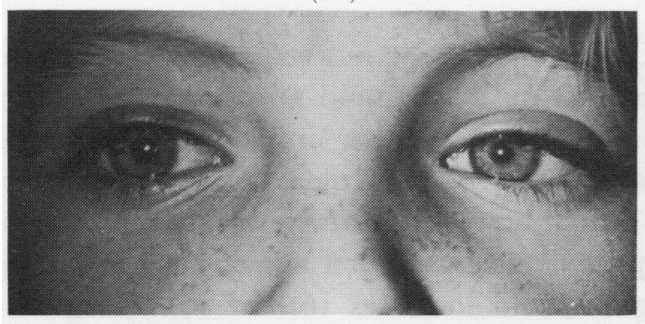

FIG. 2 Case 12. Bilateral cataract treated with irido-capsular pseudophakos implantation into the right eye at the age of 5 years. Visual acuity of the operated eye 0.8 and of the non-operated eye 0.5 . Position of the eyes straight. Fusional amplitude from $-8^{\circ}$ to $+30^{\circ}$. Positive stereo tests.
$(2 \mathrm{~B})$

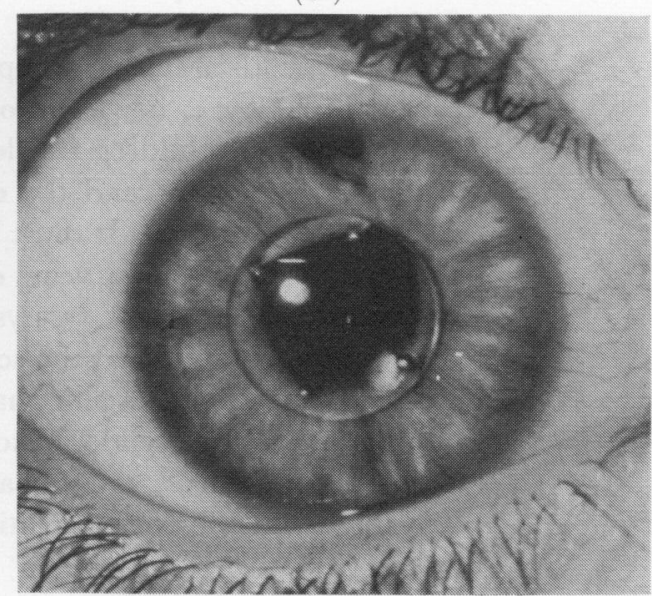

\section{Summary and conclusions}

The implantation of an irido-capsular pseudophakos is a useful procedure whereby children with congenital, infantile, or juvenile cataract can be provided with a clear pupil and approximately normal refraction. Seventeen children underwent pseudophakos implantation, fourteen in one eye and three in both, for unilateral or bilateral cataract. Their ages ranged from 8 months to i I years. No complications were observed in the follow-up period which ranged from $I \frac{1}{2}$ to $7 \frac{1}{2}$ years. The lower age limit for pseudophakic implantation is 6 months to I year. The authors' experience leads them to make the following recommendations:

(a) Pseudophakic implantation may be considered for optical reasons (nystagmus, oligophrenia), for social reasons (eye test), or for psycho-cosmetic reasons.

(b) Pseudophakic implantation is indicated in cases of imminent or recently developed squint as well as in bilateral and unilateral cataract. It is the best way of providing the squinting eye with better visual acuity and a good chance of fusion. It facilitates visual training and makes occlusion more acceptable to both child and parents. Surgery should usually be performed at a single session. 
(3) Pseudophakic implantation is strongly indicated in cases of bilateral cataract with binocular vision but insufficient acuity. Unilateral implantation into the worse eye provides the child with the required visual acuity and helps to prevent breakdown of fusion. Surgery should be performed in a single session. Implantation into the other eye is not indicated unless a squint develops.

\section{References}

BINKHORST, c. D. (1967) Brit. 7. Ophthal., 51, 767 and GoBin, м. н. (I964) Ophthalmologica (Basel), 148, I69

- - (1967) Ibid., 154, 8I

$-\longrightarrow,-$ - 